\title{
Identification of the necessary data elements to report AIDS: a systematic review
}

\author{
Mostafa Shanbehzadeh ${ }^{1}$, Maryam Ahmadi ${ }^{2}$
}

${ }^{1}$ Ph.D. Student of Health Information Management, Department of Health Information Management, School of Health Management and Information Science, Iran University of Medical Science, Tehran, Iran

${ }^{2} \mathrm{Ph}$.D. of Health Information Management, Professor, Department of Health Information Management, School of Health Management and Information Science, Iran University of Medical Science, Tehran, Iran

Type of article: Systematic review

\begin{abstract}
Background: The main focus of the surveillance system of AIDS in Iran is on direct clinical care for HIV, and other supportive facilities and secondary programmes have been somewhat neglected. Creating templates for reporting AIDS in Iran that can fulfil the needs of all information stakeholders, is a necessity for the Public Health Information Exchange (PHIE) system.

Objective: The aim of this study was to develop a comprehensive reporting template of the PHIE system for HIV/AIDS in Iran.

Methods: This study was performed in 2017. Through a systematic review of the Web of Science, Science Direct, Scopus, PubMed databases and Google Scholar search engine from 2000 to 2016, the necessary data elements are identified by the main keywords of the "Minimum Data Set" and HIV / AIDS collection.

Results: As many as 28 studies were enrolled in the research. The MDS was designed for the following categories: 1) administrative, managerial, and policy-makers (with 10 data classes and 90 data elements), 2) Clinical and medical (six data classes and 83 data elements), and 3) support services, counselling and subsidiary (three data classes and 32 data elements).

Conclusion: Iran stands in a fortunate position in terms of clinical and care programmes for AIDS treatment. A fundamental challenge is the insufficient attention to support and consulting programmes as well as the lack of information for accurate and efficient policy and decision-making. A reporting format has been designed to meet the needs of all information requirements of beneficiaries related to HIV/AIDS care and management.

Keywords: HIV /AIDS, Minimum Data Set, Health Information Exchange, Public Health
\end{abstract}

\section{Introduction}

Acquired Immune Deficiency Syndrome (AIDS), tuberculosis, and malaria are known as the original hazardous diseases for public health (1). Due to its devastating impact on the community, AIDS has recently come under the spotlight (2). The complex and multidimensional nature of this disease has made its management difficult (3). Centre for Disease Control and Prevention (CDC) of the United States has offered to use integrated information systems to tackle this disease more efficiently (4). In this regard, creating complex and coordinated templates for recording and reporting data on the disease in the form of a Standard Minimum Data Set (MDS) is an essential prerequisite for the integrated systems of information exchange on AIDS (4, 5). A workgroup comprising of global informatics medical specialists, clinical specialists of AIDS, and decision and policy-makers has achieved a consensus about the necessity of creating the MDS templates to manage and monitor treatment programmes for AIDS in developing countries (6). The MDS is a standard method to gather, store and distribute key and standard data elements (7). Creating and designing systematic MDS is one of the first and most basic steps in the creation and foundation of the national information system and improving the communication between individuals and

\section{Corresponding author:}

Professor Dr. Maryam Ahmadi, Department of Health Information Management, School of Health Management and Information Science, Iran University of Medical Science, Tehran, Iran.

Tel: +98.9122979752, Fax: +98.2188883334, Email: m_ahmadi24@yahoo.com

Received: March 04, 2017, Accepted: July 23, 2017, Published: December 2017

iThenticate screening: July 10, 2017, English editing: September 23, 2017, Quality control: November 14, 2017

This article has been reviewed / commented by Three experts

(C) 2017 The Authors. This is an open access article under the terms of the Creative Commons Attribution-NonCommercialNoDerivs License, which permits use and distribution in any medium, provided the original work is properly cited, the use is non-commercial and no modifications or adaptations are made. 
organizations involved in the care (8). The MDS is not only limited to particular clinical and care affairs of AIDS but is also used to facilitate managerial decision and policy-making aspects, research aspects, performance management, quality and financial improvement and reimbursement (6). Iran, like many other countries, has a strategic national plan to control and manage AIDS (9). However, the prevalence of AIDS is still high $(2,10,11)$ and the effective control and management process for this disease is facing many challenges $(2,9)$. One of the most important challenges is the insufficient attention to supportive and consulting programmes, and lack of information (especially accurate statistics) for decision and policy-makings (2). In one of the paragraphs of the national strategic programmes against AIDS in Iran, it emphasized on the creation of an integrated structure to collect, register, analyze and integrate distribution of information among all individuals, groups and relevant organizations (12). Thus, creating a monolithic and comprehensive framework, which considers the information needs of all beneficiaries, is necessary.

\section{Material and Methods}

In order to identify essential data elements for reporting information on clinical and managerial aspects of AIDS, a systematic review approach was used. The study was performed in 4 steps: 1) Designing and implementing search strategies, 2) Collection of related articles by inclusion or exclusion criteria, 3) Selection of final related articles, and 4) Content analysis of information.

\subsection{Design and implementing search strategies}

This study was carried out in 2017. The keywords presenting the MDS application of AIDS that were implemented in HIV/AIDS EMR, surveillance, registry and data base systems, were used in order to carry out the search. Required keywords were selected from the official PubMed website using the Mesh database and then several databases such as Web of Science, ScienceDirect, Scopus, PubMed, and Google Scholar search engine were searched based on the associated techniques of each database (Table 1). By selecting the search option in the title, abstract and keywords of the Scopus and Science Direct database, title and abstract in PubMed, and subject in Web of Science database and Google Scholar search engine, and finally implementing the input and output criteria and using the advanced search option, 25, 38, 22, 38, and 98 articles were selected as the final articles from the named databases, respectively. In Table 1, the systematic search strategy is proposed in a search field format for each of the databases.

Table 1. Search strategy details

\begin{tabular}{|l|l|}
\hline $\begin{array}{l}\text { Name of } \\
\text { Database }\end{array}$ & Search Fields \\
\hline Scopus & $\begin{array}{l}\text { ABS-KEY ("Minimum Data Set" ) OR TITLE-ABS-KEY ("Core Data Set ") OR TITLE-ABS-KEY } \\
\text { ("Minimum Data Element ") OR TITLE-ABS-KEY ("Essential Data Set ") OR TITLE-ABS-KEY } \\
\text { ("Essential Data Element ") OR TITLE-ABS-KEY ("Core Data Element ") AND TITLE-ABS-KEY } \\
\text { (AIDS) OR TITLE-ABS-KEY (HIV) AND TITLE-ABS-KEY (Report*) AND (LIMIT-TO } \\
\text { (LANGUAGE, "English" )) AND (LIMIT-TO (2000- 2016)) }\end{array}$ \\
\hline $\begin{array}{l}\text { Science } \\
\text { Direct }\end{array}$ & $\begin{array}{l}\text { TITLE-ABS-KEY ("Minimum Data Set" OR "Core Data Set" OR "Minimum Data Element" OR "Core } \\
\text { Data Element" OR "Essential Data Set " OR "Essential Data Element") and TITLE-ABS-KEY (HIV OR } \\
\text { AIDS) and TITLE-ABS-KEY (Report*) AND LANGUAGE: (English), limited to 2000- 2016. }\end{array}$ \\
\hline $\begin{array}{l}\text { Web of } \\
\text { Science }\end{array}$ & $\begin{array}{l}\text { TC=("Minimum Data Set" OR "Core Data Set" OR "Minimum Data Element" OR "Core Data Element" } \\
\text { OR "Essential Data Set " OR "Essential Data Element") AND TC=(HIV OR AIDS) AND TC=( } \\
\text { Report*) AND LANGUAGE: (English), limited to 2000- 2016. }\end{array}$ \\
\hline PubMed & $\begin{array}{l}\text { (((((((((((("Minimum Data Set "[Title/ Abstract]) OR "Core Data Set "[ Title/ Abstract]) OR "Core Data } \\
\text { Element "[ Title/ Abstract]) OR "Minimum Data Element "[ Title/ Abstract]) OR "Essential Data Set "[ } \\
\text { Title/ Abstract]) OR "Essential Data Element "[ Title/ Abstract]) AND HIV [Title/ Abstract]) OR AIDS } \\
\text { [Title/ Abstract]) AND Report* [ Title/ Abstract]))) AND (English[lang]), limited to 2000- 2016. }\end{array}$ \\
\hline $\begin{array}{l}\text { Google } \\
\text { Scholar }\end{array}$ & $\begin{array}{l}\text { Minimum Data Set " OR " Core Data Set " OR "Minimum Data Element" OR “Core Data Element" OR } \\
\text { "Essential Data Set" OR "Essential Data Element" AND "AIDS" OR "HIV" AND report* AND } \\
\text { English[lang], limited to 2000- 2016. }\end{array}$ \\
\hline
\end{tabular}

\subsection{The inclusion/exclusion criteria}

This research includes all full-text articles extracted from reliable sources in English between the years 2000 and 2016. Short articles, letters to the editor, accepted papers in conferences and reports extracted from blogs were not included in this study. The main criterion for selection of research articles was the content association of the articles with the research title. Due to the limited number of available research articles on the MDS of AIDS, several criteria 
were considered for selecting articles that have introduced necessary data elements for reporting AIDS. So, after searching with the keywords and the implementation of inclusion and exclusion criteria, titles with at least three of the following data classes related to the main objectives of reporting of AIDS were selected: 1 . Medical and clinical (primary objectives), 2. Administrative and managerial (secondary objectives) and 3. Supportive purposes (primary and secondary objectives). A search of resources and evaluation of the articles findings resulted in data enrichment such that all categories, classes, and data elements in the resources were available. Thus, there is no need for further contact with the authors of the articles.

\subsection{Choosing related articles to enter the study}

A total number of 223 articles were selected and entered into the EndNote software. After duplicates were eliminated, 112 articles were obtained. By examining titles, 58 were selected as relevant to our study and after reviewing the abstracts and the theses, 28 articles were selected for inclusion into the study, as demonstrated in Figure 1.

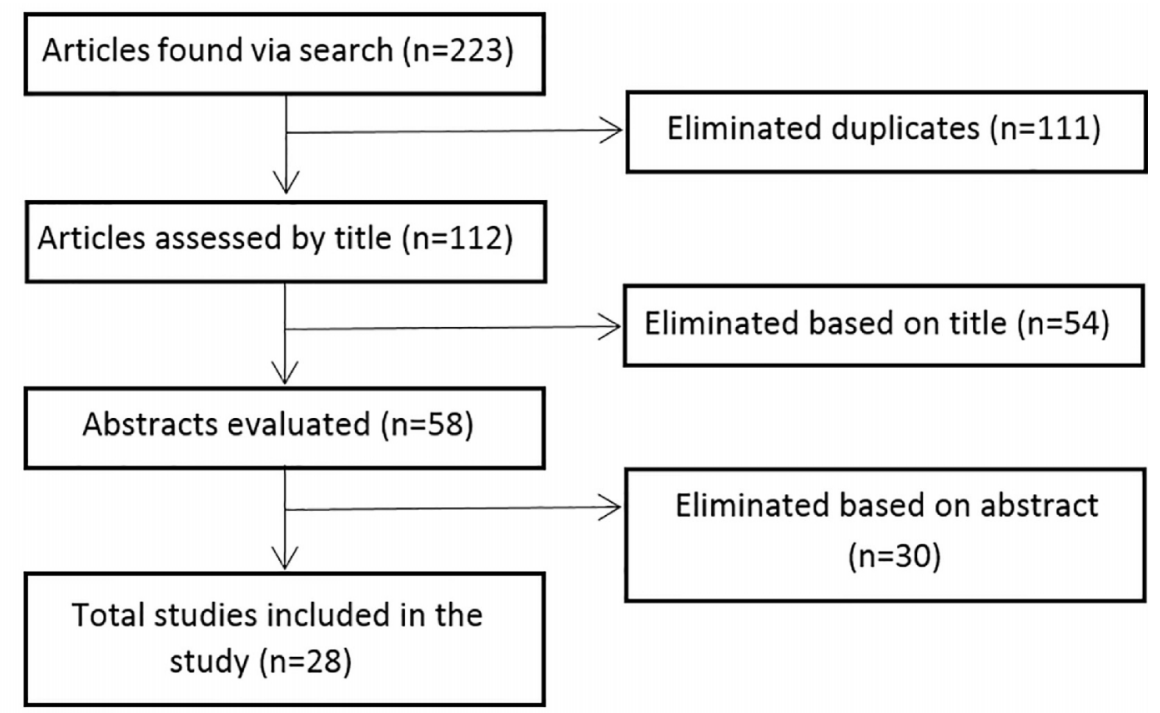

Figure 1. Article selection process in the studied databases

\subsection{Research Tools}

In this study, in order to extract data elements required for reporting AIDS from related articles, a data extraction checklist was used, which included the following categories: titles of articles, the authors' names, years of publication, article sources (including journals, conference presentation), and the method of the study. In addition, it contained a column for presenting research findings, in which the ones that contained at least three essential data classes for reporting AIDS with primary or secondary objectives were included. The form was designed by the researcher and was also based on research objectives that were used after the confirmation of its content validity by three experts. The research variables in this study are divided into three general groups including data categories (general level), data classes (detailed level) and data elements (atomic level). Among the collection of retrieved data, those articles were chosen which had at least one data class for each of the three-fold data categories. By evaluating the articles, each of the three-fold data levels above were extracted and categorized and with regard to the input and output criteria, they were entered into the data extraction form.

\subsection{Analysis of results}

In order to analyze the data, content analysis was used, which is a method to identify, analyze and report themes in the text, and has great applications in analysis of qualitative data (13).

\section{Results}

As many as 28 papers were included in the present study, five of them were directly about the MDS of AIDS, six about the EMR of AIDS, nine articles were about the surveillance systems of AIDS, one about the database of AIDS, three articles were about public health reporting systems and four about the clinical document exchange formats (e.g. Clinical Document Architecture [CDA] and Continue Care Document [CCD]) of AIDS. Figure 2 
categorises the studies by subjects. In the following, a summary of results obtained from the systematic review in 3.2. Section (Table 2) along with identified information classes and data elements in 3.3. section (Tables 3-5) is put forth. The results classified data classes and data elements necessary for HIV reporting system in three general categories: 1) Management, administrative and policy-making purposes (Table 3), 2) Medical and clinical purposes (Table 4), and 3) Supporting purposes (primary and secondary) (Table 5). Demographic information was the most frequent data class for the systemic reporting of AIDS for administrative, management and policy-making purposes that were mentioned in 23 articles. Among the demographic data elements, patient name/surname, age, sex and race were the most frequent items. In the field of financial and reimbursement reporting, data elements of the method of payment (patient self-pay, financial intermediaries, charities and other payment sources) $(6,14,29,36)$ and the type of insurance coverage (public, private) $(6,14,29,32,36)$ were of great importance. Unpopular sexual orientation (gay, unbridled/multiple sex, unsafe sex and transsexualism) $(18,29-31,39)$, repeated transfusions of blood and blood products $(18,29-31)$, and alcoholism and drug abuse $(6,19,29-31)$ were three of the most important data elements in identifying vulnerable groups and determining types of disease transmissions, which can improve the process of managing and controlling the disease. Socio-economic information was another important class for the MDS of AIDS. Among its subgroups, factors such as type of occupation as there is more exposure to diseases in certain jobs (healthcare providers, hairdressers, immigrant workers) $(16,17,19,20,38)$ and marital status (single, married, widowed or separated) $(16,19-21,23,29,33,39)$ were also introduced as important data elements in AIDS reporting processes. The importance of privacy and confidentiality on the AIDS information exchange is higher than that of many other diseases. One of the most important parts in any reporting system is its legal aspect. Receiving consent from patients is critical, especially in the field of the disclosure of information. In this regard, the data element of the confidentiality code(17) and the profile of the authorized sender and recipient $(27,28,32)$ are important. Another effective element to maintain the privacy and confidentiality of the information in patient reports is the data elements of unique identifiers. $(19,20,23,27,28,40)$. AIDS-specific tests (HIV antibody, Cluster of Differentiation 4 (CD4) and Cluster of Differentiation 8 (CD8) counts, and viral load testing) (6, 17, 20, 22, 26-28, $30,35,37)$ are the most important data elements for reporting laboratory status of AIDS. AIDS-related drug administrations (Anti Retrovirus Therapy (ART), Anti Retrovirus ARV (prevention) and Co-trim oxazole [prevention]) $(6,19-22,26,29,30)$ are essential in reporting conditions associated with administration. In many studies, the importance of determining the class of AIDS (four classes set by WHO) $(6,20-22,27,30,31,34,37$, 39 ) is emphasized in order for reporting. Information class of the patient's history is also necessary for identifying the data elements of AIDS MDS from two aspects of the clinical (primary goals) and the nonclinical (secondary goals). For example, data elements of history of disease (related or unrelated to AIDS) $(3,6,20,21,23,27,28,34$, 39 ) are important for reporting with the primary care purposes, and social history (social class, chaos, leaning towards drugs) $(20,27,28,36)$ are important for reporting with the secondary care purposes. Determining the disposition of the patient is an important data class for improving the treatment plans, among which the referral/transfer information (type, cause, date and place before or after referral/transfer( $(6,20,21,25,26,28,31$, $33,38,40)$, admission/discharge information (type, cause, date, place before or after admission/discharge) $(6,24$, $26,34,36$ ), as well as the location of the patient's referral/ transfer (hospital, emergency, specialized clinics, medical offices, counselling/support, outpatient services and public health organizations) $(18,39)$ are data elements necessary for identifying the MDS of AIDS. Consultation with the primary objectives $(17,20,34,40)$ which is directly related to care and treatment, and consultation with secondary objectives $(20,34,40)$ that indirectly affect the care and treatment, are of the most prominent data elements of reporting with supportive purposes.

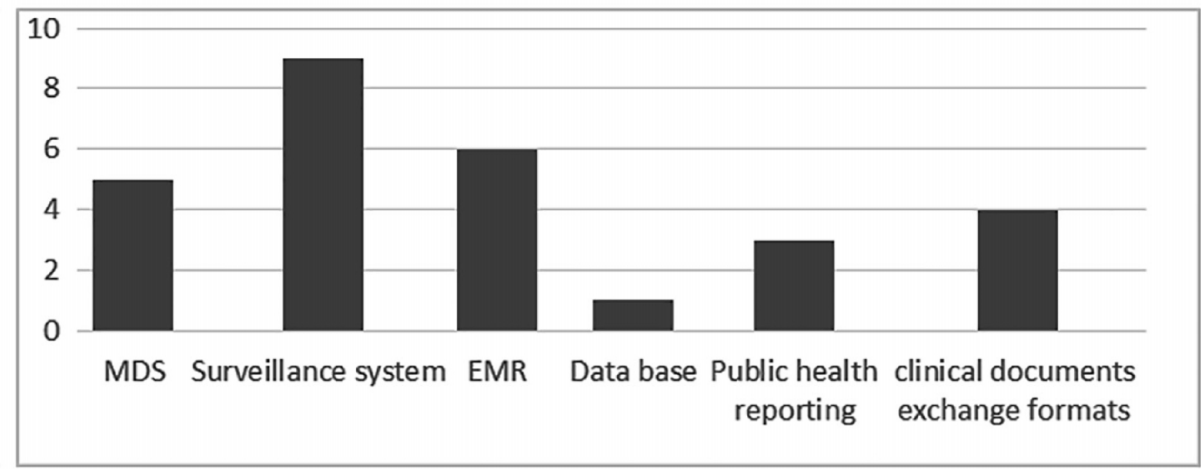

Figure 2. Categories of studies based on the research subject 
Table 2. Characteristics and results of final studies entering the research

\begin{tabular}{|c|c|c|}
\hline $\begin{array}{l}\text { Ref. } \\
\text { no. }\end{array}$ & Method & Finding \\
\hline 14 & Analysing admission assessments & $\begin{array}{l}\text { Sociodemographic characteristics, health status measures and special } \\
\text { treatments or procedures received }\end{array}$ \\
\hline 18 & $\begin{array}{l}\text { Information were abstracted from } \\
\text { AIDS cases reported in the United } \\
\text { States }\end{array}$ & $\begin{array}{l}\text { Demographic, Diagnostic, Supportive and Consulting, Laboratory, } \\
\text { Comorbidities, Prescriptive, History, Survival status, transmission } \\
\text { category and High risk groups Data Sets }\end{array}$ \\
\hline 16 & $\begin{array}{l}\text { Analysis of AIDS Case Report and } \\
\text { Incidence Trend }\end{array}$ & Sociodemographic specifications and Clinical or health status \\
\hline 17 & Descriptive Approach & Sociodemographic, Administrative, Clinical and Supportive Data Sets \\
\hline 18 & $\begin{array}{l}\text { Assessment USA AIDS Case Reports } \\
(1981-2002)\end{array}$ & $\begin{array}{l}\text { Sociodemographic, Diagnostic, High risk or vulnerable groups and } \\
\text { Utilization Healthcare Data Sets }\end{array}$ \\
\hline 19 & $\begin{array}{l}\text { Medical documentation and AIDS } \\
\text { Reports forms review }\end{array}$ & $\begin{array}{l}\text { Sociodemographic, Identification, Clinical or Diagnostic and High risk } \\
\text { or vulnerable groups Data Sets }\end{array}$ \\
\hline 12 & Cross sectional Cohort Study & $\begin{array}{l}\text { Demographic, Socio-economic, Identification, Diagnostic, Supportive } \\
\text { and Consulting, Laboratory, Prescriptive, History, Survival status and } \\
\text { Pregnancy status Data Sets }\end{array}$ \\
\hline 6 & Expert panel & $\begin{array}{l}\text { Demographics, History, Physical examination, Laboratory: HIV } \\
\text { specific, Laboratory: HIV monitoring and Imaging Data Sets }\end{array}$ \\
\hline 21 & Cohort Analysis & $\begin{array}{l}\text { Demographic, HIV care and family status, ART summary and Patient } \\
\text { encounter Data Sets }\end{array}$ \\
\hline 22 & $\begin{array}{l}\text { Data sets was derived from AIDS data } \\
\text { base }\end{array}$ & $\begin{array}{l}\text { Demographic, Encounter, Diagnostic, Laboratory, drug regimens, } \\
\text { health history, laboratory, clinical history and transmission category } \\
\text { Data Sets }\end{array}$ \\
\hline 23 & $\begin{array}{l}\text { Literature review and Survey } \\
\text { methodology }\end{array}$ & $\begin{array}{l}\text { Patient identifier, Patient contact, Problems of the patient, Responsible } \\
\text { organization or personnel Data Sets }\end{array}$ \\
\hline 24 & Content Analysis & $\begin{array}{l}\text { Patient demographics, location, clinical, Diagnostic, Medications, } \\
\text { Subject, Healthcare provider and Reporting Data Sets }\end{array}$ \\
\hline 25 & Survey methodology & $\begin{array}{l}\text { Patient demographics, diagnoses, prescription, and lab test results Data } \\
\text { Sets }\end{array}$ \\
\hline 26 & $\begin{array}{l}\text { Information were captured by the } \\
\text { Guyana's national HIV patient } \\
\text { monitoring system }\end{array}$ & $\begin{array}{l}\text { Patient chart, Pre-ART patient register and ART patient register Data } \\
\text { Sets }\end{array}$ \\
\hline 27 & Case study & $\begin{array}{l}\text { Demographic, identifier, Contact, document and Clinical or Diagnostic } \\
\text { Data Sets. }\end{array}$ \\
\hline 28 & $\begin{array}{l}\text { Data Sets was Derived from the AIDS } \\
\text { Standard guidelines and knowledge } \\
\text { bases }\end{array}$ & $\begin{array}{l}\text { Demographic information, HIV care and family status, ART summary } \\
\text { and patient encounter Data Sets }\end{array}$ \\
\hline 29 & $\begin{array}{l}\text { Data Sets was Retrieved from the } \\
\text { united states surveillance and Registry } \\
\text { systems }\end{array}$ & $\begin{array}{l}\text { Demographic, Socio-economic, Identification, Administrative and } \\
\text { policy, Clinical and Diagnostic, Supportive and Consulting, Laboratory } \\
\text { tests, Prescriptive, History, Survival status and Comorbidities Data } \\
\text { Sets }\end{array}$ \\
\hline 30 & Internet Report & $\begin{array}{l}\text { Demographics, Eligibility (Financial and Insurance), Medical (Basic, } \\
\text { History and Pregnancy), Medication, Risk Assessment and Services } \\
\text { Data Sets }\end{array}$ \\
\hline 31 & Descriptive study & $\begin{array}{l}\text { Demographic, Medical, Screening, Complementary and Warning } \\
\text { conditions Data Sets }\end{array}$ \\
\hline 32 & $\begin{array}{l}\text { Qualitative (Content analysis and } \\
\text { thematically analysis) and quantitative } \\
\text { (descriptive and multivariate statistics) } \\
\text { methods }\end{array}$ & $\begin{array}{l}\text { Member Details, Healthcare and Care Coordination Providers, } \\
\text { Medications, Result, Payer and Document Data Sets }\end{array}$ \\
\hline 33 & $\begin{array}{l}\text { Combination of qualitative (Content } \\
\text { analysis) and quantitative (survey) } \\
\text { methods }\end{array}$ & $\begin{array}{l}\text { Demographic, Clinical and Diagnostic, History, Laboratory and } \\
\text { Imaging Procedure, medications }\end{array}$ \\
\hline 34 & Survey methodology & $\begin{array}{l}\text { Patient education and referrals Data Sets, Patient clinic registration, } \\
\text { Patients inactivation, Visit registration and triage, Monitoring, } \\
\text { Appointment scheduling, Lab, Counselling and Prescription Data Sets }\end{array}$ \\
\hline
\end{tabular}




\begin{tabular}{|c|c|c|}
\hline 35 & $\begin{array}{l}\text { Check the contents of Molecular HIV } \\
\text { Surveillance system in United States }\end{array}$ & $\begin{array}{l}\text { Demographic, identifier, Clinical, History, Laboratory and } \\
\text { transmission category Data Sets }\end{array}$ \\
\hline 36 & Public health Case Report Analysing & $\begin{array}{l}\text { Demographic, identifier, Contact, Diagnostic, History, Laboratory, } \\
\text { Prescriptive, financial, Procedures, Immunization and Specimen Data } \\
\text { Sets }\end{array}$ \\
\hline 37 & $\begin{array}{l}\text { Evaluation the national HIV viral load } \\
\text { testing }\end{array}$ & $\begin{array}{l}\text { Demographic, Clinical, Laboratory, History and transmission category } \\
\text { Data Sets }\end{array}$ \\
\hline 38 & $\begin{array}{l}\text { Combination of qualitative and } \\
\text { quantitative methods }\end{array}$ & Demographic, identifier, Contact, Diagnostic and Clinical Data Sets \\
\hline 39 & $\begin{array}{l}\text { Review of the Sexually transmitted } \\
\text { diseases surveillance system in United } \\
\text { States }\end{array}$ & Demographic, clinical and behavioural data Sets \\
\hline 40 & Survey methodology & $\begin{array}{l}\text { Patients demographics, historical and physical findings, health risk } \\
\text { behaviours, tests, diagnoses and treatments Data Sets. }\end{array}$ \\
\hline
\end{tabular}

Table 3. Necessary Data classes and elements for reporting with management, administrative and policy-making purposes

\begin{tabular}{|c|c|}
\hline $\begin{array}{l}\text { Information } \\
\text { classes }\end{array}$ & Data elements / References \\
\hline $\begin{array}{l}\text { Demographic } \\
\text { information class }\end{array}$ & $\begin{array}{l}\text { 1- Name }(6,19,20,23-25,28,29,31,33,37,38,40), 2 \text {-Surname }(6,19,20,22,23,25,28,29,31, \\
33,37,38,40), 3 \text { - Father's name }(20,30), 4 \text { - Partner/spouse name }(38), 5 \text { - Age }(6,14,19,23,25, \\
26,33,35,37-39), 6 \text { - Sex }(6,14,18,19-23,25,26,29,31,35,37-39), 7 \text { - Race/ethnicity }(6,15,18, \\
20,23,25,29-31,33,35,39,40), 8 \text { - Nationality }(6,18,20,23,25,29-31,33,35,39), 9 \text { - Date of } \\
\text { birth }(6,18,21-23,30,31,35,37,38), 10 \text { - Place of birth }(6,18,21,22,23,30,31,35)\end{array}$ \\
\hline $\begin{array}{l}\text { Contact } \\
\text { information class }\end{array}$ & $\begin{array}{l}\text { 1-Address }(6,23,26,27,30,31,36,38,39), 2 \text {-Contact number }(20,23,25,30,31,36), 3 \text { - } \\
\text { Postcode/ Zip code }(23,29,30,36), 4 \text { - Fax number }(36), 5 \text {-Email address }(29,30)\end{array}$ \\
\hline $\begin{array}{l}\text { Socio-Economic } \\
\text { information class }\end{array}$ & $\begin{array}{l}\text { 1- Occupation }(16,17,20,22-25,38,39), 2 \text { - Literacy rate }(16,17,19,23,29,30,38,39), 3 \text { - } \\
\text { Health/welfare state }(16,19,29,39), 4 \text {-Type of residence }(16,35) \text {, 5-Religion }(16,19), 6 \text {-Income } \\
(16,20,23,29,39), 7-\text {-Marital status }(16,19,20,22,23,29,33), 8 \text {-Social circumstances }(19,22) \text {, } \\
\text { 10-Family status }(16,19,20,36,38), 11 \text {-Living situation }(16,17,29,35,38)\end{array}$ \\
\hline $\begin{array}{l}\text { Transmission } \\
\text { category } \\
\text { information class }\end{array}$ & $\begin{array}{l}\text { 1-Injectable drug use }(19,22,29,30,31), 2 \text {-Sexual relationship }(19,22,29,30,31,35), 3 \text {-Mother } \\
\text { to child transmission }(19,22,29,30,31,35), 4 \text {-Reception of blood and blood products }(19,22,29 \text {, } \\
\text { 30, 31), 5-Organ transplant }(19,22,26,30,31,35), 6 \text {-Intravenous injection }(19,22,29,30,31,35) \text {, } \\
\text { 7-Other categories }(19,22,35)\end{array}$ \\
\hline $\begin{array}{l}\text { High risk/at risk } \\
\text { information class }\end{array}$ & $\begin{array}{l}\text { 1-Incorrect sexual orientation }(29,30,39), 2 \text {-Receiving blood and blood products }(29,31), 3 \text { - } \\
\text { Organ transplantation }(29,31), 4 \text {-Intravascular injection }(29,31), 5 \text {-Alcohol and drug addiction }(6, \\
\text { 19, 29, 31), 6-Tattoos }(30,31), 7 \text {-Occupational hazards }(30,31), 8 \text {-Travel/ immigration }(26,29,30 \text {, } \\
\text { 31), 9-Mental disease }(30), 10 \text { - Other risks }(30), 11 \text {-Reason of exposure }(25), 12 \text {-Location of } \\
\text { exposure }(25), 13 \text { - Duration of exposure }(25), 14 \text {-Date of exposure }(25), 15 \text {-activity during } \\
\text { exposure }(25)\end{array}$ \\
\hline $\begin{array}{l}\text { Patient dispo } \\
\text { information }\end{array}$ & $\begin{array}{l}\text { 1-Referral/transfer info (source, cause, date, place, type) }(6,21,22,36,40), 2 \text {-Follow-up info }(25, \\
26,31,36), 3 \text {-Admission info (source, cause, date, place, type, })(6,21,24,26,34,36), 4- \\
\text { Discharge info (source, cause, date, place, type, })(6,21,24,26,34,36), 5 \text {-Treatment plans }(27,28 \text {, } \\
33,36), 6 \text {-Recommendations at discharge }(23)\end{array}$ \\
\hline $\begin{array}{l}\text { Financial } \\
\text { information class }\end{array}$ & $\begin{array}{l}\text { 1- Payment type }(6,14,17,32), 2 \text {-Payment method (14, 17), 3-Payment source (6), 4-Policy / } \\
\text { coverage type }(6,14,29,31,36), 5 \text {-Insurance name (6, 32), 6-Insurer company (17), 7-Insurance } \\
\text { credit time (17), 8-Payer name (17), 9-covered party ID (32), 10-Member status (17) }\end{array}$ \\
\hline $\begin{array}{l}\text { Legal information } \\
\text { class }\end{array}$ & $\begin{array}{l}\text { 1- Privacy Code (17), 2- Medical/prescription errors (17) } \\
\text { Consent for the following purposes: 3-Discharge by personal desire (17), 4- Disclosure of } \\
\text { information (17) }\end{array}$ \\
\hline $\begin{array}{l}\text { Identification } \\
\text { information class }\end{array}$ & $\begin{array}{l}\text { Identification number of: 1-Patient }(6,19-24,27,33,37), 2 \text {-Medical record }(20,23,27,36), 3- \\
\text { Admission/visit }(20,27) \text {, 4-Treatment/ART therapy }(20), 5 \text { - Medical specialist }(6,19,20,22,24 \text {, } \\
\text { 26, 27, 40), 6- Provider institute }(19,20-24,27,37,40), 7 \text { - Payment }(6,17,32), 8 \text { - Specimen }(24 \text {, } \\
\text { 37), 9- Family }(6,20), 10 \text {-Social security }(6,20), 11 \text {-Referral/transfer }(20)\end{array}$ \\
\hline $\begin{array}{l}\text { Document } \\
\text { information class }\end{array}$ & $\begin{array}{l}\text { 1- Document name }(27,28,32), 2 \text { - Document title }(27,28,32), 3 \text { - Document goal }(27,28,32), 4- \\
\text { Document creation date }(27,28,32), 5 \text {-Document author }(27,28,32), 6 \text { - profile of the authorised } \\
\text { transmitter/receiver }(27,28,32), 7 \text { - Document description }(27,28,32)\end{array}$ \\
\hline
\end{tabular}


Table 4. Necessary Data classes and elements for reporting with clinical and medical purposes

\begin{tabular}{|c|c|}
\hline Information classes & Data elements / References \\
\hline $\begin{array}{l}\text { Diagnostic } \\
\text { information class }\end{array}$ & $\begin{array}{l}\text { 1- Diagnosis finding (related or unrelated to AIDS) }(6,14,19,20,27-29,34,39), 2 \text {-primary and } \\
\text { final diagnosis }(6,20,23,24,27-29,31,39), 3 \text { - HIV status (active, Inactive, recovered) }(6,20, \\
\text { 21, 36, 39), 4- test/diagnosis method }(20,24,36), 5 \text { - Chief Complaint }(6,21,22,24,27,28,34), \\
6 \text {-Signs and Symptoms }(14,19-24,27,28,30,34,37,39,40), 7 \text { - Physical examination }(6,14,20 \text {, } \\
\text { 24, 27, 28, 33, 40), 8- Assessment \& Plan }(20,24,27,28), 9 \text { - Body Mass Index }(19,20,27,28, \\
\text { 38), 10-HIV staging (WHO classes) }(6,20-22,27,28,30,37,39), 11 \text {-HIV subtype (type one or } \\
\text { two }(6,14,15,20,21,27,28), 12 \text { - Vital Signs }(6,21-23,34), 13 \text { - medical procedures (related or } \\
\text { unrelated to AIDS) }(6,40), 14 \text {-first sign/symptom onset date }(23,24), 15 \text { - HIV diagnosis date } \\
(23,31,35,37), 16 \text { - age of patient at the time of diagnosis }(18), 17 \text {-the duration between being } \\
\text { infected and the diagnosis }(16,18)\end{array}$ \\
\hline $\begin{array}{l}\text { Prescription/ } \\
\text { pharmaceutical } \\
\text { information class }\end{array}$ & $\begin{array}{l}\text { General prescription: } 1 \text { - Prescription name }(6,19-22,29,30,32,36), 2 \text {-Drug form }(21,30,32 \text {, } \\
\text { 36), 3-Consumption dose }(21,30,32,36), 4 \text {-Consumption timing }(21,30,32,36), 5 \text {-Route of } \\
\text { administration }(21,30,32,36) 6 \text {-Drug's target tissue }(21,30,32,36), 7 \text { - Prescription date }(21, \\
\text { 30, 32, 34, 35), } 8 \text { - Drug allergy }(17,20,21,23,34), 9 \text {-Drug toxicity }(17,20,21,23,34), 10 \text { - } \\
\text { Drugs adverse effects }(17,20,21,34) \text {. Anti-Retrovirus Therapy / Anti Retrovirus (ART/ARV) } \\
\text { summary: } 1 \text {-current ART/ARV regime }(20,21,25,26,34,37), 2 \text {-ART/ARV eligibility (date, } \\
\text { reason) }(20,21,25,26,34,37), 3 \text {-ART/ARV initiation (date, reason) }(20,21,25,26,34,37,38) \text {, } \\
\text { 4-ART/ARV end (date, reason) }(20,21,25,26,34,37), 5 \text {-ART/ARV discontinuation/ } \\
\text { intermittent (date, reason })(17,20,21,25,26,34,37), 6 \text { - ART/ARV alternative /changing (date, } \\
\text { reason) }(20,21,25,26,34,37), 7 \text {-ART/ARV dispense (date, reason) }(20,21,25,26,34,37), 8- \\
\text { time between diagnosis and the ART initiation }(16,20,21,25,26,34,37), 9 \text { - HIV stage at ART } \\
\text { initiation }(20,21,25,26,34), 10 \text {-CD4 and CD8 levels when starting the ART }(20,25,26,34), \\
\text { 11- Compliance/adherence assessments }(6,16,20,21,37,38)\end{array}$ \\
\hline $\begin{array}{l}\text { Laboratory } \\
\text { information class }\end{array}$ & $\begin{array}{l}\text { 1- HIV test name }(6,20-22,24,27,28,30,31,35,37), 2 \text {-HIV test type }(6,20,21,24,31,36), 3- \\
\text { Test normal range/reference }(20,24,32), 4 \text { - Frequency of testing }(20,32), 5 \text { - First HIV test date } \\
(20,30,32,39), 6 \text { - Related (sub) tests }(20-22,32), 7 \text { - HIV test result }(6,21,32), 8 \text { - Date of first } \\
\text { HIV test }(20,32,35), 9 \text {-Date of first positive HIV test outcome }(20,32,35,39), 10 \text { - Date of last } \\
\text { negative HIV test outcome }(20,32), 11 \text {-HIV/AIDS diagnosis date }(20,32), 12 \text { - Radiographic } \\
\text { information }(6,30,33,40), 13 \text {-Specimen data (gathering date }(24,36,37) \text {, type }(24,36,37) \text {, } \\
\text { quality }(24,36,37) \text {, temperature }(24,36,37) \text {, the date of receipt }(24,36,37) \text {, interpretation date } \\
(24,36,37) \text {, result date }(20,24,32,35,36,37)\end{array}$ \\
\hline $\begin{array}{l}\text { History information } \\
\text { class }\end{array}$ & $\begin{array}{l}\text { 1- Diseases/condition history }(6,20,21,23,27,28,34,39), 2 \text { - Medication history }(\text { ART/non } \\
\text { ART) }(6,20,21,27,28,40), 3 \text { - Procedure/ treatment history }(6,20,27,28,40), 4 \text { - High-risk } \\
\text { sexual relationship history }(6,20,26), 5 \text {-Pregnancy/delivery history }(20), 6 \text {-Mental history }(20 \text {, } \\
27,28,40), 7 \text {-Social history }(20,28,36)\end{array}$ \\
\hline $\begin{array}{l}\text { Comorbidities/ } \\
\text { warning diseases } \\
\text { information class }\end{array}$ & $\begin{array}{l}\text { 1- Tuberculosis }(20,29,30,38), 2 \text {-Hepatitis }(18,20,29,30), 3 \text { - Sexually transmitted infections } \\
(20,22,29,30), 4 \text {-Cancer }(18,20,29,30), 5 \text { - Pneumonia }(18,20,29,30), 6 \text { - Opportunistic } \\
\text { infections }(14,18,20,29,30,33,35), 7 \text {-other }(20,29,30)\end{array}$ \\
\hline $\begin{array}{l}\text { Survival } \\
\text { information class }\end{array}$ & $\begin{array}{l}\text { 1- The current state of life }(6,14,20,29), 2 \text {-Underlying cause of death }(6,14,20,29), 3 \text {-Date of } \\
\text { death }(6,14,20,29), 4 \text { - The time between diagnosis and death }(16,20), 5 \text {-The time between } \\
\text { initiation of the ART until death }(16,20)\end{array}$ \\
\hline
\end{tabular}

Table 5. Necessary Data classes and elements for reporting with supportive purposes

\begin{tabular}{|l|l|}
\hline Information classes & Data elements / References \\
\hline $\begin{array}{l}\text { Consulting program } \\
\text { information class }\end{array}$ & $\begin{array}{l}\text { Consult: 1- Behavioral (17, 20, 34), 2- Family/family planning (17, 20, 34), 3-Pregnancy / } \\
\text { delivery (17, 20, 29, 34), 4-Nutrition and diet therapy (17, 20, 34), 5- Rehab (20, 29, 34), 6- } \\
\text { Addiction (20, 34), 7-Occupational (17, 20, 34), 8-Prevention (29, 34), 9-Medication/timing of } \\
\text { consumption (20,34), 10-Before and after the first test (34), 11-Religious (34), 12-Marital (34), } \\
\text { 13-Sexual behaviors (34) }\end{array}$ \\
\hline $\begin{array}{l}\text { Support programs } \\
\text { information class }\end{array}$ & $\begin{array}{l}\text { 1-Charities (29), 2-Education and awareness programs (29), 3-Housing assistance (29), 4- } \\
\text { Transportation assistance (29), 5-Isolation (26, 29), 6-screening (20, 30, 31, 39), 7-Social care } \\
\text { services (17, 29), 8-Advocacy groups (29, 31), 9-Treatment supporter (29-31) }\end{array}$ \\
\hline $\begin{array}{l}\text { Subsidiary services } \\
\text { information class }\end{array}$ & $\begin{array}{l}\text { 1-Laboratory services (40), 2-Imaging procedures (40), 3-Dental care (40), 4-mother/baby health } \\
\text { (40), 5-Rehabilitation services (29, 40), 6-Immunization services (20, 27-30, 36, 40), 7- } \\
\text { Preventive or prophylaxis services (29), 8-Contraceptive management (39, 40), 9-antenatal care } \\
\text { (40) }\end{array}$ \\
\hline
\end{tabular}




\section{Discussion}

\subsection{The horizon ahead for Iran}

The reasons behind why the prevalence of AIDS is high in Iran is the country being located beside the biggest narcotic transportation in the world in neighbouring Afghanistan and the high incidence of the disease in its northern neighbour - Turkmenistan (2). Additionally, in recent years, an increase in the amount of infection by sexual contact (especially among youngsters) has also fuelled this problem (11). The foundation of reporting systems of conditions associated with AIDS in Iran is known as one of the basic requirements to control and manage this disease (10). The PHIE systems in the form of bilinear reporting systems, among all beneficiaries involved in control and management of AIDS disease at local and national level, can play a great role in the inhibition of this disease (9). A study by Lai et al. (2008) showed that the use of the MDS of AIDS improves care by effective information exchange, and changes the traditional interactions between the teams involved in the care (41). The integrated AIDS care system consists of various parts of the health care industry including hospitals and other healthcare provider organizations, supportive and subsidiary service providers, public health, government, legislators, policy-makers and reimbursement agencies (6). Providing a comprehensive MDS that can meet the needs of all individuals and associated groups is the essential prerequisite of an integrated system of AIDS information exchange (42, 6). AIDS treatment and care programmes in Iran offer a good level of services, but the challenge lies in the insufficient attention to supportive and counselling programmes, as well as the lack of information for making an accurate and efficient decision and policy (2). These deficiencies should be considered in the design of the MDS of integrated data reporting of AIDS, and the needs of all the involved groups should be considered as unified $(6,2)$. The coverage of proposed data elements, as reviewed in the studies, are generally limited to medical and diagnostic domains, and the needs of secondary care, supportive and counselling activities have been rarely considered. For example, Tierney et al. (2006) have prioritised the needs of care professionals (doctors, nurses and paramedics) to the needs of managers and administrative staff, agencies and legislatures, policy-makers, reimbursement agencies, researchers and support institutions for the design of the MDS of AIDS) (6, 40). In Iran, due to the specific social, cultural and religious characteristics, people often think of AIDS as a stigma caused by sex outside marriage (11); Most patients in this situation will not be willing to undergo tests and follow their treatment, and they will not be notified until the progression and appearance of the symptoms of disease (2). The exact prevalence rate of AIDS is unclear in Iran due to long concealment of the disease because of the mass reluctance to carry out consultations and diagnostic tests (10). The disease has also profound effects on the physical, mental, spiritual, family and job functions, as well as the social and economic statuses of individuals (43); So, there is a greater need for protective measures, counselling programmes, awareness and education (especially before and after the first test, at the time of positive result and during the treatment process) (2). In this study, one of the three goals of reporting AIDS is related to support. Data classes, associated with it, are crafted in three parts: consulting, supportive and subsidiary services. Health, social, economic, and demographic statuses affect the pattern of AIDS in countries. Social participation, the physical and mental performance of individuals, disability (physical or mental), social security services, and immigration status can be useful for monitoring and evaluation system of AIDS (44). In a study conducted by Morgan (2012), social records such as employment status, marital status, lifestyle, moral status, leaning towards drugs and alcohol, housing and protection system (guardian) are mentioned as necessary data elements for facilitating policy and decision-making processes (29). In this study, in addition to the application of data elements for the improvement of healthcare (primary objective), the secondary needs (administrative, management, and policy-making) have also been considered. Hence, the decision and policy-making activities against AIDS in Iran can be improved with the elimination of the available information shortage $(45,2)$. Therefore, in the designed MDS, an attempt was made to consider the existing deficiencies in a comprehensive care programme for AIDS in Iran (especially in the secondary objectives of care). In addition, a comprehensive data set has been provided for medical and diagnostic procedure and support programmes, in order for continuous and patient-oriented treatment.

\subsection{The strengths of the suggested MDS}

Mirzaee et al. (2013) examined the types of HIV transmission in large surveillance systems to identify at-risk groups in Iran (46). The present study has considered the data elements of transmission and at-risk groups, important for care and policy-making objectives (Table 3). In two studies conducted by Patra et al. (2010) (2011), on the creation of CDA for AIDS, data elements were structured in two parts: CDA title (meta information about the document) and on the other hand, CDA body (narrative information) $(27,28)$. In the present study, offering MDS in two categories of nonclinical data classes (demographic, identification and document information classes) for CDA title and clinical (diagnostic, prescriptive, laboratory, history) for CDA body, has become a necessary prerequisite for CDA template designing (Tables 3, 4). About $80 \%$ of the cases, monitored for epidemic HIV disease in the community, need laboratory interventions (47). One of the most important technological infrastructures, used in public health reports, 
is the use of an Electronic Laboratory Reporting (ELR) system. The basic prerequisite for this infrastructure is the complete and standard definition of laboratory data elements (48). In the present study, laboratory data are introduced as the most important elements for reporting AIDS, and it was planned to be designed in such a way that the ELR needs could be met (Table 4). One of the challenges facing the early diagnosis of patients with AIDS in Iran and persuading them to actively participate in the management of their treatment, is fear of information disclosure and honour endangerment (2). Thus, in the current study, another attempt was made to consider the requirement of confidentiality and security of information in the design of the MDS. This requirement has been met via the legal information class (different consents and codes of confidentiality of information), document information class (profile of the authorized receiver and transmitter) and unique identification information class (Table 3). Rosen et al. (2016) evaluated the information of the ART prescription, including scheduling, assessments of adherence, qualifying the receipt and history of it regarding the level of CD4. These two were introduced as complementary to each other for advanced analyses (49). The role of prescribing information, especially the ART, was great for reporting in this study (Table 4). Palla et al. (2006) reviewed the cause of death due to AIDS (direct and indirect). They noticed over time a change in the pattern of the cause of death due to AIDS (50). In the present study, this obligation was fully predicted in the overall category of survival status information class (Table 3 ).

\subsection{The weaknesses of the proposed MDS}

Determining the type of antiretroviral treatment, failure can be defined as a less than desirable response to treatment. These types of failures include virology, immunology, and clinical failure. Reporting this situation in the AIDS reports is useful for clinical evaluations (51). Unfortunately, in the current study, none mentioned this information class. Elements of the pregnancy status in HIV reporting include information of previous pregnancy/pregnancy history, date of last menstrual period, estimated delivery time, gestational age, pregnancy/gestational planning and contraception $(20,34,40)$ required to be evaluated in a separate data class for the medical/clinical purposes in this study.

\section{Conclusions}

The designed MDS in the present study considers the HIV reporting system in three main categories: 1 . Medical and clinical, 2. Management, administrative, and policy-making, and 3. Consulting, supportive, and subsidiary services. The designed data framework makes it possible to overcome the problems of the comprehensive system of AIDS treatment and management in Iran. However, its prerequisite, and standardizing content and the structure of data messages in the form of messaging protocols in the network for optimum interoperability, is essential.

\section{Acknowledgments:}

This article was part of a Ph.D. dissertation supported by the Iran University of Medical Sciences (Grant no: IUMS/SHMIS/2016/9221563207).

\section{Conflict of Interest:}

There is no conflict of interest to be declared.

Authors' contributions:

All authors contributed to this project and article equally. All authors read and approved the final manuscript.

\section{References:}

1) Vitoria M, Granich R, Gilks CF, Gunneberg C, Hosseini M, Were W, et al. The global fight against HIV/AIDS, tuberculosis, and malaria. Am J Clin Pathol. 2009; 131(6): 844-8. doi: 10.1309/AJCP5XHDB1PNAEYT. PMID: 19461091.

2) Mohammadpour A, Yekta ZP, Nasrabadi ARN, Mohraz M. Coming to terms with a diagnosis of HIV in Iran: a phenomenological study. J Assoc Nurses AIDS Care. 2009; 20(4): 249-59. doi: 10.1016/j.jana.2009.03.003. PMID: 19576542.

3) Myers JJ, Koester KA, Chakravarty D, Pearson C, Maiorana A, Shade SB, et al. Perceptions regarding the ease of use and usefulness of health information exchange systems among medical providers, case managers and non-clinical staff members working in HIV care and community settings. Int J Med Inform. 2012; 81(10): e21-e9. doi: 10.1016/j.ijmedinf.2012.07.005. PMID: 22854159.

4) Shade SB, Chakravarty D, Koester KA, Steward WT, Myers JJ. Health information exchange interventions can enhance quality and continuity of HIV care. Int J Med Inform. 2012; 81(10): e1-e9. doi: 10.1016/j.ijmedinf.2012.07.003. PMID: 22854158. 
5) Zabat G, Caoili J, Anduyon A, Ramos G, Morin M, Ditangco R, et al. Design and implementation of an HIV/AIDS electronic medical record system (HIV/AIDS EMR) in the Philippines. AMIA Annu Symp Proc. 2007: 1166. PMID: 18694262.

6) Tierney WM, Beck EJ, Gardner RM, Musick B, Shields M, Shiyonga NM, et al. Viewpoint: A Pragmatic Approach to Constructing a Minimum Data Set for Care of Patients with HIV in Developing Countries. J Am Med Inform Assoc. 2006; 13(3): 253-60. doi: 10.1197/jamia.M2005.

7) Ahmadi M, Alipour J, Mohammadi A, Khorami F. Development a minimum data set of the information management system for burns. Burns. 2015; 41(5): 1092-9. doi: 10.1016/j.burns.2014.12.009. PMID: 25561018.

8) Ahmadi M, Mohammadi A, Chraghbaigi R, Fathi T, Baghini MS. Developing a minimum data set of the information management system for orthopedic injuries in iran. Iran Red Crescent Med J. 2014; 16(7). doi: 10.5812/ircmj.17020. PMID: 25237576, PMCID: PMC4166095.

9) Khajekazemi R, Rezazadeh M, Mhzari K. Islamic Republic of Iran AIDS Progress Report. National AIDS Committee Secretariat. Ministry of Health and Medical Education. 2015. Available from: http://www.unaids.org/sites/default/files/country/documents/IRN_narrative_report_2015.pdf.

10) Haghdoost AA, Mostafavi E, Mirzazadeh A, Navadeh S, Feizzadeh A, Fahimfar N, et al. Modelling of HIV/AIDS in Iran up to 2014. Journal of AIDS and HIV Research. 2011; 3(12): 231-9. doi: 10.5897/JAHR11.030.

11) Tavoosi A, Zaferani A, Enzevaei A, Tajik P, Ahmadinezhad Z. Knowledge and attitude towards HIV/AIDS among Iranian students. BMC Public Health. 2004; 4(1): 1. doi: 10.1186/1471-2458-4-17. PMID: 15157281, PMCID: PMC420470.

12) The third National AIDS Strategic Plan. Iranian ministry of health; 2009. Available from: http://idrc.resv.hums.ac.ir/uploads/57_734_80_2.pdf.

13) Vaismoradi M, Turunen $\mathrm{H}$, Bondas T. Content analysis and thematic analysis: Implications for conducting a qualitative descriptive study. Nurs Health Sci. 2013; 15(3): 398-405. doi: 10.1111/nhs.12048. PMID: 23480423.

14) Buchanan RJ, Wang S, Huang C. Analyses of nursing home residents with HIV and dementia using the minimum data set. Journal of acquired immune deficiency syndromes (1999). 2001; 26(3): 246-55. doi: 10.1097/00042560-200103010-00006.

15) AIDS Public Information Data Set. Washington, DC: US Department of Health and Human Services; 2000: $1-52$.

16) Castilla J, Sobrino P, de la Fuente L, Noguer I, Guerra L, Parras F. Late diagnosis of HIV infection in the era of highly active antiretroviral therapy: consequences for AIDS incidence. Aids. 2002; 16(14): 1945-51. doi: 10.1097/00002030-200209270-00012. PMID: 12351955.

17) Fraser HS, Jazayeri D, Nevil P, Karacaoglu Y, Farmer PE, Lyon E, et al. An information system and medical record to support HIV treatment in rural Haiti. BMJ. 2004; 329(7475): 1142-6. doi: 10.1136/bmj.329.7475.1142. PMID: 15539669, PMCID: PMC527691.

18) AIDS Public Information Data in the United States for the years 1981-2002. United States: Centers for Disease Control and Prevention; 2016.

19) Siika AM, Rotich JK, Simiyu CJ, Kigotho EM, Smith FE, Sidle JE, et al. An electronic medical record system for ambulatory care of HIV-infected patients in Kenya. Int J Med Inform. 2005; 74(5): 345-55. doi: 10.1016/j.jimedinf.2005.03.002. PMID: 15893257.

20) World Health Organization, UNICEF. Three interlinked patient monitoring systems for HIV care; 2012.

21) Patient monitoring guidelines for HIV care and antiretroviral therapy. WHO; 2006. Available from: http://www.who.int/3by5/capacity/ptmonguidelinesfinalv1.PDF.

22) Altuğlu I, Cavuşoğlu C, Ciçek C, Tünger O. Development of a database for tracking HIV positive/AIDS patients. Mikrobiyol bul. 2007; 41(1): 101-8. PMID: 17427558.

23) Liu D, Wang X, Pan F, Xu Y, Yang P, Rao K. Web-based infectious disease reporting using XML forms. Int J Med Inform. 2008; 77(9): 630-40. doi: 10.1016/j.ijmedinf.2007.10.011. PMID: 18060833.

24) Rajeev D, Staes CJ, Evans RS, Mottice S, Rolfs R, Samore MH, et al. Development of an electronic public health case report using HL7 v2. 5 to meet public health needs. J Am Med Inform Assoc. 2010; 17(1): 3441. doi: 10.1197/jamia.M3299. PMID: 20064799, PMCID: PMC2995632.

25) Were MC, Shen C, Bwana M, Emenyonu N, Musinguzi N, Nkuyahaga F, et al. Creation and evaluation of EMR-based paper clinical summaries to support HIV-care in Uganda, Africa. Int J Med Inform. 2010; 79(2): 90-6. doi: 10.1016/j.ijmedinf.2009.11.006. PMID: 20036193, PMCID: PMC2818680. 
26) Halpern M, Lachmansingh B, Minior T, Hasbrouck LM, Persaud N, Foo A. Implementation of a standardized HIV patient monitoring system in Guyana. Rev Panam Salud Publica. 2010; 28(2): 107-1. doi: 10.1590/S1020-49892010000800006. PMID: 20963277.

27) Patra D, Mukhopadhyay J, Majumdar A, Majumdar B, Dogra D. Tele-consultation using Clinical Document Architecture in disease specific domains. e-Health Networking Applications and Services. 12th IEEE International Conference on; 2010.

28) Patra D, Mukherjee J, Majumdar A, Majumdar B, Das Bhattacharya S, Paul S. A Standard, Knowledge Integrated Consultation Document for Pediatric HIV Information Exchange. Journal of Healthcare Engineering. 2011; 2(2): 161-82. doi: 10.1260/2040-2295.2.2.161.

29) Ford MA, Spicer CM. Monitoring HIV care in the United States: indicators and data systems: National Academies Press; 2012.

30) HIV Care Program (HCP) Minimum Data Set. 2012. Available from: https://www.cdph.ca.gov/programs/aids/Documents/ARIESHCPMinimumDataset.pdf.

31) Nematollahi M, Moghaddasi H, Askarian M, Afsarkazerooni P. National HIV/AIDS Surveillance Pattern for Iran's Health System. Health Information Management. 2008; 4(2): 153-66.

32) Gordon P, Camhi E, Hesse R, Odlum M, Schnall R, Rodriguez M, et al. Processes and outcomes of developing a continuity of care document for use as a personal health record by people living with HIV/AIDS in New York City. Int J Med Inform. 2012; 81(10): e63-e73. doi: 10.1016/j.ijmedinf.2012.06.004. PMID: 22841825, PMCID: PMC4447096.

33) Schnall R, Cimino JJ, Bakken S. Development of a prototype continuity of care record with contextspecific links to meet the information needs of case managers for persons living with HIV. Int J Med Inform. 2012; 81(8): 549-55. doi: 10.1016/j.ijmedinf.2012.05.002. PMID: 22632821, PMCID: PMC3515778.

34) Castelnuovo B, Kiragga A, Afayo V, Ncube M, Orama R, Magero S, et al. Implementation of providerbased electronic medical records and improvement of the quality of data in a large HIV program in SubSaharan Africa. PloS One. 2012; 7(12): e51631. doi: 10.1371/journal.pone.0051631. PMID: 23284728, PMCID: PMC3524185.

35) Technical Guidance for HIV Surveillance Programs Molecular HIV Surveillance (MHS). Atlanta, Georgia: HIV Incidence and Case Surveillance Branch; 2012. Available from: http://www.reginfo.gov/public/do/DownloadDocument?objectID=65853001.

36) Standards \& interoperability framework public health reporting initiative data harmonization profile Version 2.0. U.S: Health and Human Services Office of the National Coordinator for Health IT; 2013.

37) Technical and operational considerations for implementing HIV viral load testing. Geneva, Switzerland: WHO; 2014.

38) Puttkammer NH. Using Data from the iSanté National Electronic Medical Record System to Strengthen Haiti's HIV Antiretroviral Therapy Program. University of Washington; 2014.

39) Enhanced STD Surveillance Network (eSSuN) Protocol and Project Implementation Guide; 2015. Available from: https://www.cdc.gov/std/ssun/protocol_v8.2_508.pdf.

40) Tierney WM, Sidle JE, Diero LO, Sudoi A, Kiplagat J, Macharia S, et al. Assessing the impact of a primary care electronic medical record system in three kenyan rural health centers. J Am Med Inform Assoc. 2015: ocv074. doi: 10.1093/jamia/ocv074. PMID: 26260246.

41) Lai TY, Larson EL, Rockoff ML, Bakken S. User Acceptance of HIV TIDES - tailored interventions for management of depressive symptoms in persons living with HIV/AIDS. J Am Med Inform Assoc. 2008; 15(2): 217-26. doi: 10.1197/jamia.M2481.

42) Magnus M, Herwehe J, Gruber D, Wilbright W, Shepard E, Abrams A, et al. Improved HIV-related outcomes associated with implementation of a novel public health information exchange. Int J Med Inform. 2012; 81(10): e30-e8. doi: 10.1016/j.ijmedinf.2012.06.005. PMID: 22883431.

43) Fabiani M, Cawthorne A, Nattabi B, Ayella EO, Ogwang M, Declich S. Investigating factors associated with uptake of HIV voluntary counselling and testing among pregnant women living in North Uganda. AIDS Care. 2007; 19(6): 733-9. doi: 10.1080/09540120601087731. PMID: 17573592.

44) Kowal P, Peachey K. Indicators for the minimum data set project on ageing: a critical review in subSaharan Africa. Dar es Salaam, United Republic of Tanzania: WHO; 2001: 9.

45) Eshrati B, Asl RT, Dell CA, Afshar P, Millson PM, Kamali M, et al. Preventing HIV transmission among Iranian prisoners: initial support for providing education on the benefits of harm reduction practices. Harm Reduction Journal. 2008; 5(1): 21. doi: 10.1186/1477-7517-5-21. 
46) Mirzaei M, Poorolajal J, Khazaei S, Saatchi M. Survival rate of AIDS disease and mortality in HIVinfected patients in Hamadan, Iran: A registry-based retrospective cohort study (1997-2011). Int J STD AIDS. 2013; 24(11): 859-66. doi: 10.1177/0956462413486457. PMID: 23970604.

47) Rajeev D, Staes CJ, Evans RS, Price A, Hill M, Mottice S, et al. Evaluation of HL7 v2. 5.1 electronic case reports transmitted from a healthcare enterprise to public health. AMIA Annu Symp Proc. 2011; 2011: 1144-52. PMID: 22195175, PMCID: PMC3243216.

48) Rose D. The Value of Electronic Laboratory Reporting for Public Health. Scientific Technologies Corporation. 2010.

49) Rosen S, Fox MP, Larson BA, Sow PS, Ehrenkranz PD, Venter F, et al. Accelerating the Uptake and Timing of Antiretroviral Therapy Initiation in Sub-Saharan Africa: An Operations Research Agenda. PLoS Med. 2016; 13(8): e1002106. doi: 10.1371/journal.pmed.1002106. PMID: 27505444 PMCID: PMC4978457.

50) Palella Jr FJ, Baker RK, Moorman AC, Chmiel JS, Wood KC, Brooks JT, et al. Mortality in the highly active antiretroviral therapy era: changing causes of death and disease in the HIV outpatient study. J Acquir Immune Defic Syndr. 2006; 43(1): 27-34. doi: 10.1097/01.qai.0000233310.90484.16. PMID: 16878047.

51) Van Oosterhout JJ, Brown L, Weigel R, Kumwenda JJ, Mzinganjira D, Saukila N, et al. Diagnosis of antiretroviral therapy failure in Malawi: poor performance of clinical and immunological WHO criteria. Trop Med Int Health. 2009; 14(8): 856-61. doi: 10.1111/j.1365-3156.2009.02309.x. PMID: 19552661. 